\title{
CPT® (Common Procedural Terminology) - A Primer for Neuroradiologists
}

\author{
C Segovis ${ }^{1}$, H Valand ${ }^{2}$ R Bhala ${ }^{3}$, Gicola $^{4}$, W Donovan ${ }^{5}$, R Tu$^{6}$ \\ ${ }^{1}$ Emory School of Medicine, Johns Creek, GA \\ ${ }^{2}$ American University of Integrative Sciences, Tucker, GA \\ ${ }^{3}$ ASNR, Oakbrook, IL \\ ${ }^{4}$ Hackensack Radiology Group, Hackensack, NJ \\ ${ }^{5}$ Hartford HealthCare, New Haven, CT \\ ${ }^{6}$ Progressive Radiology, Washington, DC
}

Purpose:

CPT® (Common Procedural Terminology) is the system of medical nomenclature used to report procedures and services to public and private health insurance programs. This code set affects any neuroradiologist billing for services in the United States. Understanding how CPT® codes are created and how the code set is maintained will help neuroradiologists be more effective in the reimbursement process.

\section{Approach/Methods:}

An interactive, visually-oriented education presentation designed to educate neuroradiologists on the importance of $\mathrm{CPT} \circledast$ in the process of appropriate reimbursement, and the role of ASNR in the process of code creation and code set maintenance.

\section{Education Objectives:}

- Demonstrate the role of $\mathrm{CPT} 尺$ in the reimbursement process.

- Demonstrate the different types of codes within the CPT® code set and how these different codes relate to neuroradiology practice.

- Gain insight into code creation and code set maintenance, and demonstrate the role of ASNR in this process.

\section{Summary/Conclusion:}

Understanding how CPT $®$ codes are created and how the code set is maintained will help neuroradiologists be more effective in the reimbursement process.

\section{Categories:}

HEALTH POLICY, Clinical Practice

\section{References:}

1. CPT® overview and code approval. https://www.ama-assn.org/practice-management/cpt/cpt- overview-and-codeapproval Accessed October 31, 2019

2. The CPT® code process. https://www.ama-assn.org/about/cpt-editorial-panel/cpt-code-process. Accessed November 1,2019

3. Thorwarth WT. From concept to CPT code to compensation: how the payment system works. J Am Coll Radiol 2004; 1(1): $48-53$ 


\section{What is a CPT $®$ Code?}

Common Procedural Terminology or CPT $®$ is the system of standardized medical nomenclature used to report medical, surgical, and diagnostic services.

The code set was formally adopted for procedural reporting under the Health Insurance Portability and Accountability Act (HIPAA).

The American Medical Association (AMA) owns and maintains the code set.

\section{What is the structure of a CPT® code?}

A CPT $®$ code is a five-digit code (numeric or alphanumeric) that is used to describe a service.

Each code and relevant descriptor is listed in the $\mathrm{CPT} \otimes$ code manual

For example, a head CT without contrast:

$70450=$ Computed tomography, head or brain, without contrast material

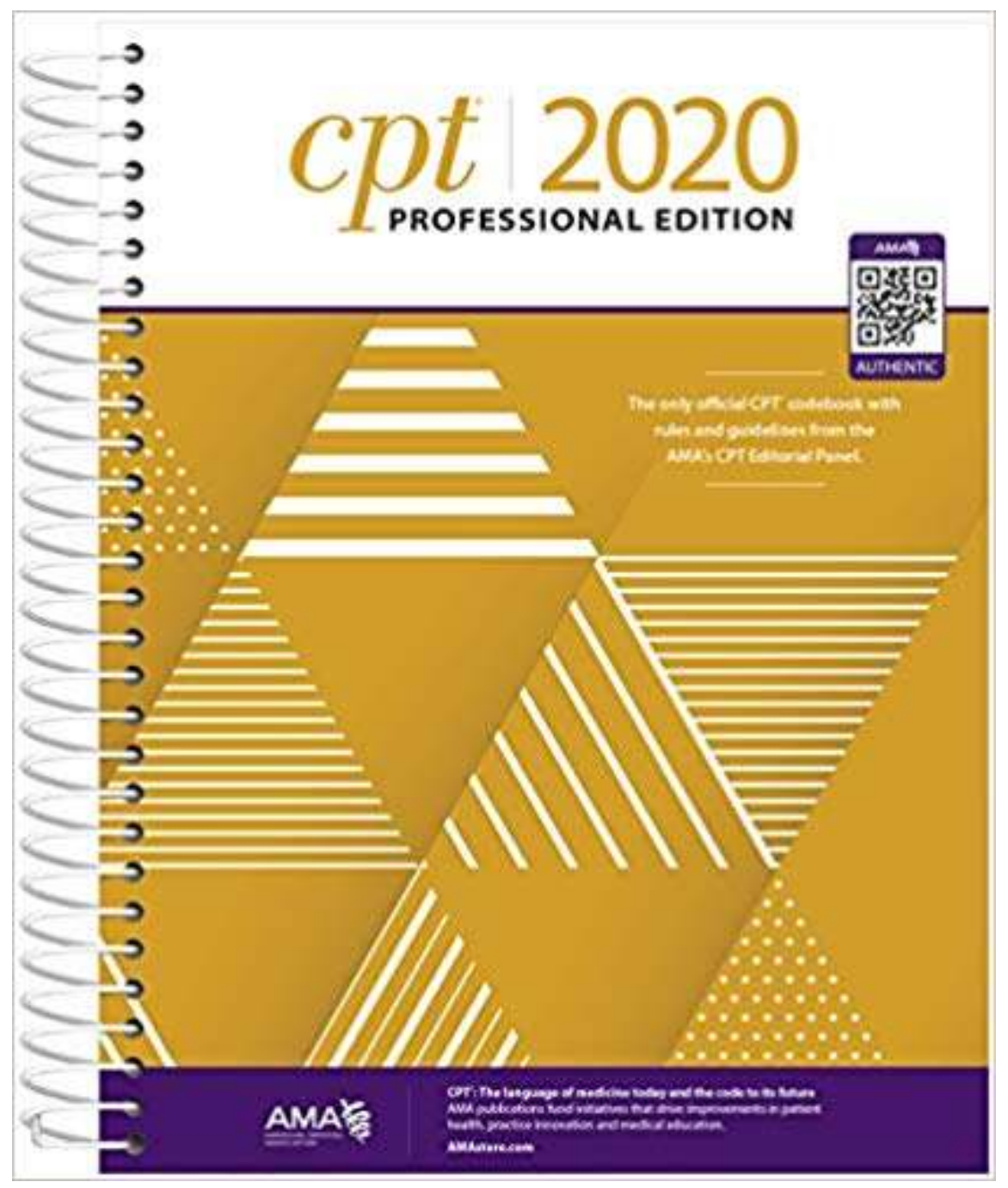

Why is CPT® relevant to your practice?

A CPT® code is what is reported to a payor to describe the work you do. 


\section{What are the types of CPT® Codes?}

\section{Category I}

Codes describing widely used diagnostic services and procedures, consistent with contemporary medical practice, performed by many practitioners and multiple locations, and approved by the FDA when appropriate.

The class of codes that make up the bulk of the code set.

Example: $70450=$ Computed tomography, head or brain, without contrast material

\section{Category II}

Supplemental alphanumeric tracking codes used for performance measurement.

Category II codes are not required for correctly coding a procedure or service.

Category II codes are used to track performance metrics. These codes can be used as QDCs (Quality Data Code) in Merit-based Incentive Payment System (MIPS).

Example: $3100 \mathrm{~F}=$ Carotid imaging study report (included direct or indirect reference to measurement of distal internal carotid diameter as denominator for stenosis measurement)

\section{Category III}

Temporary alphanumeric codes for new and developing technology, procedures and services.

These codes were created for data collection, assessment and in some instances, payment of new services and procedures that currently do not meet the criteria for a Category I code.

Example: $0042 \mathrm{~T}=$ Cerebral perfusion analysis using computer tomography with contrast administration, including post-processing of parametric maps with determination of cerebral blood flow, cerebral blood volume, and mean transit time. 


\section{What are CPT® Code Modifiers?}

A modifier is a mechanism to indicate that the reporting provider has altered a service or procedure.

Modifiers are indicated by a numeric code added to the based code. For example, 70450-26. Examples of CPT® code modifiers include:

\section{Modifier - Professional Component}

The 26 modifier is used to indicate billing of only the professional component of a service.

\section{Modifier - Reduced Services}

A service or procedure that the provider chooses to cancel prior to service completion or that has been completed but the completed service is less than a defined CPT $®$ code.

\section{Modifier - Discontinued Procedure}

A service or procedure that is terminated because the patient is at risk, and appropriate medical practice indicated termination of the procedure.

\section{Modifier - Distinct Procedural Service}

Procedures or services, other than E/M services, that are not normally reported together, but are appropriate to report together under the circumstances.

Application of modifiers can be complex. Your coders, billing company, or experts should be consulted for guidance. 


\section{Who manages the CPT® Code Set?}

\section{The CPT® Editorial Panel}

The CPT® Editorial Panel is a group of 17 individuals tasked by the AMA Board of Trustees to "to revise, update, or modify CPT codes, descriptors, rules and guidelines."

The individuals on the CPT $®$ panel include:

- 11 physicians nominated by national medical specialty societies.

- A physician from each of the following groups: Blue Cross and Blue Shield Association, America's Health Insurance Plans, American Hospital Association, CMS

- 2 members from the CPT® Health Care Professionals Advisory Committee.

\section{CPT® Advisors and the CPT® Advisory Committee}

CPT® Advisors are primary physicians who are volunteer their time and are nominated by national medical specialty societies seated in AMA House of Delegates. Volunteer representatives also come from the AMA Health Care Professionals Advisory Committee (HCPAC) which represent limited-license practitioners and other allied health professionals.

The representatives serve on the CPT $®$ Advisory Committee. The purpose of the committee is to serve as a resource to the CPT $®$ Editorial Panel, AMA staff, and to the members of the AMA and component medical societies. Advisors' roles include:

- Advising on procedure coding and appropriate nomenclature as relevant to the member's specialty

- Suggesting revisions to the CPT® code set.

- Provide documentation to staff and the CPT® Editorial Panel regarding the medical appropriateness of various medical and surgical procedures under consideration for inclusion in the CPT $®$ code set.

- Prepare technical education material and articles pertaining to the CPT® code set.

- Promote and educate the medical community about the use and benefits of the CPT® code set. 


\section{How are CPT® codes created or revised?}

A requests for a new CPT® code or revision of an existing CPT® code occurs through submission of a code change application to the AMA. An application can be submitted by any interested party.

The CPT® Editorial Panel meets 3 times a year to review code change applications, and to solicit input from physicians, advisors from national medical societies, members of industry, and potentially the public.

The process of CPT Editorial Panel review includes review of the application and supporting materials by the panel, presentation of the code change application to the Editorial Panel by assigned panel members, a discussion of the application by the panel and interested parties (individuals who submitted the code change application, members of CPT® Advisory Committee), and a vote by the panel for action on the application. The potential outcomes of the vote include:

- Addition of a new code or revision of existing nomenclature - this change would appear in a forthcoming volume of CPT®

- Referral to a workgroup for further study

- Postponement to a future meeting (to allow submittal of additional information in a new application)

- Rejection of the item

The decision of the CPT® Editorial Panel and published after the meeting in the panel digest.

Submission of Code Change Application (new code or revision)

Review of application by AMA Staff

Review and commentary by Medical Specialty Societies on the CPT® Advisory Committee (includes ASNR)

Review of application, supporting materials, and CPT® Advisory Committee comments by CPT Editorial Panel

Application presented and discussed at CPT® Editorial Panel Meeting

CPT® Editorial Panel votes on application

Decision of CPT® Editorial Panel published in Summary of Panel Actions after the meeting. Any new codes or revision approved by the Editorial Panel are included in the forthcoming volume of the CPT® manual 


\section{Alternative mechanisms for CPT ${ }^{\circledR}$ code creation or revision}

\section{The AMA RUC Relativity Assessment Workgroup (RAW)}

CMS and RVS Update Committee (RUC) Relativity Assessment Workgroup (RAW) monitor utilization of procedures and practice. Procedures that are typically performed together are often flagged for bundling.

Bundling is not automatic. Members of the ASNR CPT® and RUC teams, both staff and physician volunteers, work closely with CMS and other stakeholders to defend current practices if appropriate and create new codes that will best help us help our patients if new codes are appropriate.

\section{Example:}

Before 2020, Lumbar puncture with fluoroscopic guidance for either therapeutic diagnostic was billed as 2 separate codes. For example, a diagnostic lumbar puncture using fluoroscopic guidance was billed as:

\section{- 62270 - Spinal puncture, lumbar, diagnostic}

- 77003- Fluoroscopic guidance and localization of needle or catheter tip for spine or paraspinous diagnostic or therapeutic injection procedures (epidural or subarachnoid)

62270 describes the work of performing the lumbar puncture and 77003 is added to describe the use of imaging guidance. 62270 is reported alone if imaging guidance is not used.

Lumbar puncture practice patterns in the US have changed. Imaging guidance is now typically used as Radiologists are now the typical provider of this service.

(10.2214/AJR.14.12622). This change in practice pattern resulted a code change request from CMS via the RAW process to create a new bundled code describing a lumbar puncture performed with image guidance.

Prior to 2020

62270

Spinal puncture, lumbar, diagnostic

77003

Fluoroscopic guidance
Start in 2020

62328

Spinal puncture, lumbar, diagnostic

With fluoroscopic or CT guidance

This new group of bundled codes also includes lumbar puncture for therapeutic purposes, 62329. MR and ultrasound guidance are not included in this bundle. 


\section{Alternative mechanisms for $\mathrm{CPT} \circledast$ code creation or revision}

\section{Nation Correct Coding Initiative (NCCl)}

The $\mathrm{NCCl}$ is a CMS initiative tasked "to promote national correct coding methodologies and to control improper coding leading to inappropriate payment in Part B claims."

There are 2 classes of edits published by the $\mathrm{NCCl}$

- Procedure-to-procedure (PTP) edits - designed to prevent improper payment when incorrect code combinations are combined

- Medical Unlikely (MUE) edits - designed to prevent services from being reported with incorrect units of service (e.g. \# of times a service is performed.)

Procedures that are potentially miscoded are flagged for clarification and/or deletion by $\mathrm{CMS}$ though $\mathrm{NCCl}$ edits. The ASRN staff and physician volunteers work closely with other members of the stakeholder community to review proposed edits by the $\mathrm{NCCl}$ process and argue against inappropriate edits that do not reflect appropriate practice patterns.

Example:

Radiographic myelography is often performed in conjunction with spine CT with intrathecal contrast (CT myelography). Prior to 2015, the NCCl flagged this combination as potentially duplicative. ASNR and other stakeholders successfully appealed to the $\mathrm{NCCl}$ that these procedures although often performed together are not duplicative and constitute a Distinct Procedural Service (DPS), indicated by Modifier 59.

The $\mathrm{NCCl}$ recommended adding the 59 modifier to the base code to clarify that these procedure may be mistaken as duplicative.

Radiographic myelography also shows another example of bundling. 62304 is a bundled code for myelography via lumbar injection, including RS\&I, that is used if a single provider performs both myelography and the associated CT myelogram.

Scenario - 2 providers performing the procedure

Physician 1 - completes injection

Physician 2-completes radiological supervision and interpretation (RS\&I)

Codes for submission:

76000 Fluoroscopy, up to $1 \mathrm{hr}$

62284 Lumbar injection

72265 Myelogram, lumbosacral, RS\&I

72132-59 CT lumbar spine with contrast (intrathecal)
Scenario - 1 provider performs the procedure

Single physician completing both injection and RS\&I

Codes for submission:

76000 Fluoroscopy, up to $1 \mathrm{hr}$

62304 Myelography via lumbar injection, including RS\&I

(BUNDLED INJECTION WITH RS\&I. 62284+72265=62304)

72132-59 CT lumbar spine with contrast (intrathecal)

This results demonstrates the hard work of ASNR staff and the physician volunteers in defending how the work we do is documented in the reimbursement cycle. 


\section{Does a CPT® code guarantee payment?}

Creation of a code is a multistep process that involves both creation of the code through the CPT® Editorial panel and valuation of the code through the RUC process.

But a $C P T \circledast$ code does not guarantee payment.

Category I codes - these code make up the bulk of the code set and describe widely used diagnostic services and procedures, consistent with contemporary medical practice, performed by many practitioners and multiple locations, and approved by the FDA when appropriate. These code are valued by the RUC process.

There are additional steps for establishing if a procedure or service will be covered by CMS through the processes of National Coverage Determination (NCD) or Local Coverage Determination (LCD).

National Coverage Determination (NCD) - the process CMS uses to determine if a service is to be coverage at a national level to CMS beneficiaries.

Local Coverage Determination (LCD) - the process used by the Medicare Administrative Contractors (MACs) to determine if a product or service is to be covered if the product or service is not covered through a NCD.

Medicare Administrative Contractor (MAC) - a private health care insurer that has been awarded rights to a geographic jurisdiction to process Medicare Part A and Part B medical claims or Durable Medical Equipment (DME) claims for Medicare Fee-ForService (FFS) beneficiaries.

Furthermore, insurance companies can decide if a CPT® code will be reimbursed for their beneficiary population.

\section{Can Category III codes be reimbursed?}

Yes.

It is incorrect to believe that Category III codes cannot be reimbursed. Although a Category III code has not undergone valuation by the RUC process, this does not mean that a Category III cannot be reimbursed. Some commercial payors will consider reimbursement of Category III codes.

MACs can also consider reimbursing Category III codes through the LCD process. 


\section{Addressing concerns about future image bundling}

Bundling imaging services with other codes remains a challenge within the CPT® set. The CPT Editorial Panel created the Imaging Bundling Workgroup to address how image bundling is integrated within the CPT® code set. ASNR is represented on this workgroup.

A goal of the workgroup is NOT to retroactively bundle in imaging into existing services

The primary goal of the workgroup is to define a set of criteria that will help set expectations and provide guidelines for both the CPT® Panel and applicants as to when and how imaging guidance should be bundled into the primary procedure/service for new and revised codes going forward.

\section{How can I assist in correct coding?}

Documentation is critical for correct coding.

Actions that radiologists can perform to assist in correct coding:

1. Verify the clinical indication is appropriate for the imaging study and if not acquire the necessary information.

2. Avoid terms such as "Concern for..., assess for..., rule out..., suspicion for..., evaluate for..." in the indication as these terms cannot be used to code a definite diagnosis.

3. Document number of views in radiography.

4. Verify the technique matches the procedure performed (e.g. was contrast used?)

5. Be careful when changing an order (e.g. MR brain with contrast to MR brain without contrast). This can result in non-payment if the exam underwent pre-certification.

6. Know your coders and help them help you! 


\section{Summary}

- Common Procedural Terminology or CPT® is the system of standardized medical nomenclature used to report medical, surgical, and diagnostic services.

- $\mathrm{CPT} \circledast$ is managed by the AMA for CMS.

- Categories

- Category I- accepted services (majority)

- Category II - supplemental performance measures

- Category III - new and emerging technologies

- Maintenance of the code set occurs through the work of AMA staff, staff of component societies such as ASNR, and numerous physician volunteers through a systematic process.

- The code set is not static with changes made to reflect current practice.

- You and your documentation are important for correct coding.

The ASNR staff and physician volunteers are working for you and your patients to ensure an appropriate reimbursement landscape.

\section{Thank you}

Questions?

colin.m.segovis@emory.edu

@colinsegovis 